\title{
Carta al editor Nitratos dietarios y su impacto en la salud humana
}

\author{
Letter to the editor \\ Dietary nitrates and its impact on human health \\ Carta ao editor \\ Nitratos dietéticos e o seu impacto na saúde humana
}

https://doi.org/10.35454/rncm.v4n1.229

Estimada editora:

Primero, queremos felicitarlos por su interesante revista. Hemos leído con gran interés el artículo publicado por Pereira y Ramírez ${ }^{(1)}$, en su sección primera en línea del 29 de octubre de 2020. Sin duda, es un artículo de suma importancia que intenta describir acertadamente la exposición, el metabolismo y la biotransformación de nitratos y nitritos. Además, analiza algunos de los efectos a la salud humana por la ingesta de estos compuestos, tanto benéficos como negativos.

Sin embargo, con el fin de precisar la información, quisiéramos añadir que, en la parte que aborda la exposición a nitratos y nitritos, únicamente existen dos vías importantes de exposición: la primera es endógena y se rige por la vía clásica de la L-arginina-óxido nítrico sintasa, implicando el metabolismo de óxido nítrico endógeno que, dada su naturaleza reactiva, se oxida rápidamente en los tejidos y el torrente sanguíneo para dar origen a sus productos más estables: nitrito y, en presencia de oxihemoglobina, nitrato. La segunda vía es exógena y es orquestada por la vía enterosalival nitrato-nitrito-óxido nítrico, utilizando principalmente nitratos y nitritos inorgánicos dietarios $^{(2)}$.

También quisiéramos aclarar que, donde se menciona la ingesta diaria aceptable de nitrato $(3,7 \mathrm{mg} / \mathrm{kg}$ de peso/ día) y nitrito $(0,07 \mathrm{mg} / \mathrm{kg}$ de peso/día) establecida por la Autoridad Europea de Seguridad Alimentaria (EFSA), se hace referencia a una ingesta que incluye no solo aditivos alimentarios, sino que incluye además a los vegetales y al agua de consumo, por lo que tal límite de ingesta diaria aceptable no es exclusivo de los vegetales. Al respecto, quisiéramos recomendar la revisión de la Tabla 1 , la cual podría ejemplificar de mejor manera las cantidades y los límites permisibles de nitratos y nitritos a través de la ingesta de vegetales ${ }^{(3)}$.

Sumado a lo anterior, en todo el documento no se observa ninguna mención sobre los efectos negativos en la función tiroidea como consecuencia de la exposición a nitratos; sin embargo, existe literatura que indica el desarrollo de hipotiroidismo ante una dieta vegana en niños ${ }^{(4)}$, siendo ellos la población más vulnerable ante dicha exposición.

Por otra parte, existe literatura que indica que los nitratos fungen como verdaderos disruptores tiroideos ${ }^{(5)}$, por lo cual nos parece de suma importancia incluir, dentro de los efectos negativos en la salud humana, a las disfunciones tiroideas causados por estos compuestos nitrogenados.

Finalmente, apreciamos los beneficios que tiene la ingesta de nitratos, sobre todo en los efectos cardiovasculares, pero hacemos hincapié en que debemos conocer los factores que están involucrados en las concentraciones de estos compuestos en los vegetales, tales como el tipo de vegetal, la estación y la localización de siembra, el almacenamiento y la manera de preparación, las practicas antropogénicas como agricultura intensiva, y la concentración de agua en su estructura, entre otros ${ }^{(3)}$, algo que en el documento pasa desapercibido.

Somos conscientes de que su labor es de divulgación y difusión de la investigación científica, pero reiteramos nuestro punto de vista sobre las omisiones que podrían resultar en una confusión y desinformación para el lector. 
Tabla 1. Comparación del consumo de nitraros y nitritos en la Unión Europea con otros países (mg/persona/día) y la ingesta diaria aceptable (ADI)

\begin{tabular}{|l|c|c|c|c|}
\hline \multicolumn{1}{|c|}{$\begin{array}{c}\text { País } \\
\text { (Referencia) }\end{array}$} & $\begin{array}{c}\text { Consumo de } \\
\text { vegetable (g/día) }\end{array}$ & $\begin{array}{c}\text { Peso corporal medio } \\
\text { del adulto (kg) }\end{array}$ & $\begin{array}{c}\text { Ingesta de nitrato } \\
\text { (mg/día) }\end{array}$ & $\begin{array}{c}\text { Consumo de } \\
\text { nitrato*** (mg/día) }\end{array}$ \\
\hline $\begin{array}{l}\text { Unión Europea } \\
\text { (EFSA, 2008) }\end{array}$ & 400 & 60 & $157-457$ & $0,2-0,8$ \\
\hline $\begin{array}{l}\text { EE. UU. } \\
\text { (Hord y colaboradores, 2009) }\end{array}$ & Dieta DASH* & 60 & $174-1222$ & $0,351-0,41$ \\
\hline $\begin{array}{l}\text { Japón } \\
\text { (Sobko y colaboradores, 2010) }\end{array}$ & $\begin{array}{l}\text { JTD** (espinaca } \\
\text { inglesa) máx. }\end{array}$ & 60 & 1128 & - \\
\hline $\begin{array}{l}\text { Australia } \\
\text { (Hsu y colaboradores, 2009) }\end{array}$ & 231 & 70 & 618 & - \\
\hline $\begin{array}{l}\text { Nueva Zelanda } \\
\text { (Thomson y colaboradores, 2007) }\end{array}$ & $\begin{array}{l}704 \\
\text { (Col china) }\end{array}$ & - & 52,5 & 0,59 \\
\hline $\begin{array}{l}\text { Corea } \\
\text { (Chung y colaboradores, 2003) }\end{array}$ & 510 & 60 & 182 & 1,12 \\
\hline $\begin{array}{l}\text { China } \\
\text { (Zhong y colaboradores, 2002) }\end{array}$ & & 60 & 222 & 0,78 \\
\hline $\begin{array}{l}\text { Valor ADI (máx.) } \\
\text { (FAO/WHO, 2003) }\end{array}$ & & & & \\
\hline
\end{tabular}

*Enfoques dietéticos para detener el patrón dietético de la hipertensión.

**Dieta tradicional japonesa

***Sin conversión de nitrato a nitrito.

DASH: Dietary Approaches to Stop Hypertension; JDT: japanese traditional diet.

Modificado de: Hmelak Gorenjak A, et $\mathrm{al}^{(3)}$

\section{Edgar García Torres}

Clínica de admisión y diagnóstico

Facultad de Odontología, Universidad Juárez del Estado de Durango. Durango, México

E-mail: smiledent.coi@hotmail.com

\section{Fernanda Elizabeth Rodríguez Rodríguez}

Clínica de admisión y diagnóstico

Facultad de Odontología, Universidad Juárez del Estado de Durango. Durango, México.

Recibido: 9 de noviembre 2020

\section{Referencias bibliográficas}

1. Pereira ML, Ramírez BDG. Nitratos y nitritos, la doble cara de la moneda. Rev Nutr Clin Metab. 2020;4(1). doi: 10.35454/ rncm.v4n1.202 [primero en línea].

2. Lundberg JO, Weitzberg E. Biology of nitrogen oxides in the gastrointestinal tract. Gut. 2012;62(4):616-629. doi:10.1136/gutjnl-2011-301649.

3. Hmelak Gorenjak A, Cencič A. Nitrate in vegetables and their impact on human health. A review. Acta alimentaria. 2013;42(2):158-172. doi: 10.1556/aalim.42.2013.2.4.
4. Brandt A, Cheung M, Sakka S, Ajzensztejn M, Hulse T. Awareness of the Risks of Acquired Iodine Deficiency in Strict Vegan Diets. ESPE Abstracts. 2018;89:P2-P411.

5. García Torres E, Pérez Morales R, González Zamora A, Ríos Sánchez E, Olivas Calderón EH, Alba Romero JJ, et al. Consumption of water contaminated by nitrate and its deleterious effects on the human thyroid gland: a review and update. Int J Environ Health Res. 2020;1-18. doi: 10.1080/09603123.2020.1815664 [en prensa]. 